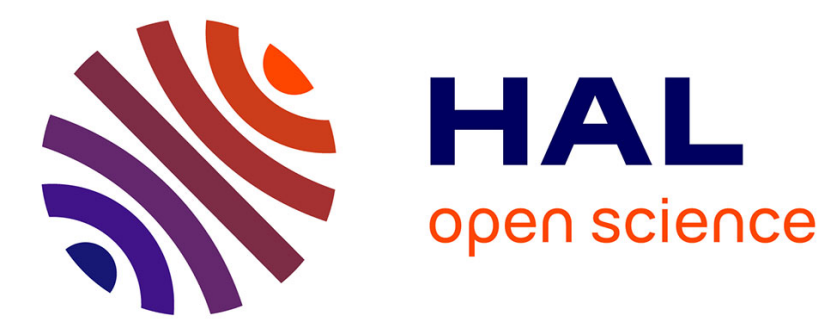

\title{
Coherent and paracoherent states in Josephson-coupled granular superconductors
}

\author{
J. Rosenblatt
}

\section{To cite this version:}

J. Rosenblatt. Coherent and paracoherent states in Josephson-coupled granular superconductors. Revue de Physique Appliquée, 1974, 9 (1), pp.217-222. 10.1051/rphysap:0197400901021700 . jpa00243742

\section{HAL Id: jpa-00243742 https://hal.science/jpa-00243742}

Submitted on 1 Jan 1974

HAL is a multi-disciplinary open access archive for the deposit and dissemination of scientific research documents, whether they are published or not. The documents may come from teaching and research institutions in France or abroad, or from public or private research centers.
L'archive ouverte pluridisciplinaire HAL, est destinée au dépôt et à la diffusion de documents scientifiques de niveau recherche, publiés ou non, émanant des établissements d'enseignement et de recherche français ou étrangers, des laboratoires publics ou privés. 


\title{
COHERENT AND PARACOHERENT STATES IN JOSEPHSON-COUPLED GRANULAR SUPERCONDUCTORS
}

\author{
J. ROSENBLATT
}

Institut National des Sciences Appliquées, 35031 Rennes Cedex, France

Centre National d'Etudes en Télécommunications, 22301 Lannion, France

\begin{abstract}
Résumé. - Le paramètre d'ordre d'un ensemble de grains supraconducteurs de diamètre plus grand que la longueur de cohérence est un ensemble de vecteurs dans le plan complexe. On montre que si les grains sont couplés par l'effet Josephson, le système est analogue à un ferromagnétique d'Heisenberg avec un espace de spin à deux dimensions. Il se produit une transition de phase d'un état paracohérent à un état cohérent à une température $T_{0}<T_{\mathrm{c}}$. En dessous de la température de cohérence $T_{0}$ les phases sont ordonnées et l'ensemble du système présente des effets Josephson. On obtient les équations de mouvement des phases et les effets des fluctuations sur les caractéristiques $V$-I. Les supraconducteurs granulaires obéissent à l'électrodynamique de London et présentent des effets d'interférence quantique particuliers.
\end{abstract}

\begin{abstract}
The order parameter of an assembly of superconducting grains of diameter greater than the coherence length is a set of vectors in the complex plane. It is shown that if the grains are coupled through the Josephson effect, the system is the analog of a Heisenberg ferromagnet with spin dimensionality 2 . A phase transition occurs from a paracoherent state to a coherent one above and below, respectively, a coherent temperature $T_{0}<T_{\mathrm{c}}$. It is only in the latter domain of temperatures, where superconducting phases are ordered, that Josephson effects will be fully displayed by the system as a whole. Approximate equations of motion are derived and thereby effects due to phase fluctuations upon the DC characteristics are studied theoretically and experimentally. The systems obey London electrodynamics and possess peculiar quantum interference properties.
\end{abstract}

1. Introduction. - We shall be concerned here with bulk granular superconductors (BGS) [1], that is, one-, two- or three-dimensional arrays of bulk superconducting regions (grains or not-toothin thin films) interconnected by weak links displaying Josephson effects. Such systems are not conceptually different from those described in the preceding paper [2]. In fact, our samples look typically as in figure 1, differing from Dr. Clark's in grain diameter $(\simeq 170 \mu \pm 10 \mu$, which allowed us to have a few hundred contacts per $\mathrm{mm}^{3}$ ) and in their threedimensional character. The main interest of such systems is of course the possibility of superradiance [3], which stems from a physical phenomenon which one can call synchronisation, that is, locking of phase slip velocities in neighbouring links interacting through an electromagnetic field. This is a dynamical effect whose efficiency may strongly depend on another feature of the system, namely phase coherence. We mean by this ordering of superconducting phases in different grains coupled by the Josephson phase dependent interaction. Phase ordering is an equilibrium property of arrays whose effects are apparent already in their DC behaviour. In the following we shall successively study the process through which phase coherence is attained, a model allowing to

REVUE DE PHYSIQUe APPLIQUÉE. - T. 9 No 1, JANVIER 1974 make definite predictions on the dc properties of BGS, and the electrodynamics of BGS in the coherent state.

2. Transition to phase coherence. - Let us point out that our experimental results [4], [5], as well as other studies [6], [7] indicate that BGS usually show Josephson behaviour only at temperatures sizeably different from the superconducting critical temperature $T_{\mathrm{c}}$. In particular the dynamical resistance at zero current bias $R=\mathrm{d} V /\left.\mathrm{d} I\right|_{I \rightarrow 0} \neq 0$ in a more or less extended temperature region. One may ascribe this to the presence of thermal noise in the system, but then the effective noise temperature which would fit the measured values of $R$ [8] decreases sharply by more than an order of magnitude around a certain bath temperature $T_{0}$, characteristic of each sample.

We have proposed the following explanation for this behaviour [5]. The superconducting order parameter of an assembly of superconducting grains in the absence of applied current can be represented by a set of vectors in the complex plane $\Delta_{\alpha}=|\Delta| \mathrm{e}^{i \varphi_{\alpha}}$, $\varphi_{\alpha}$ being the superconducting phase in the $\alpha$ th grain. For bulk grains the superconducting free energy (proportional to the volume) is much greater than $k T$ immediately below $T_{\mathrm{c}}$, so that fluctuations in $|\Delta|$ 


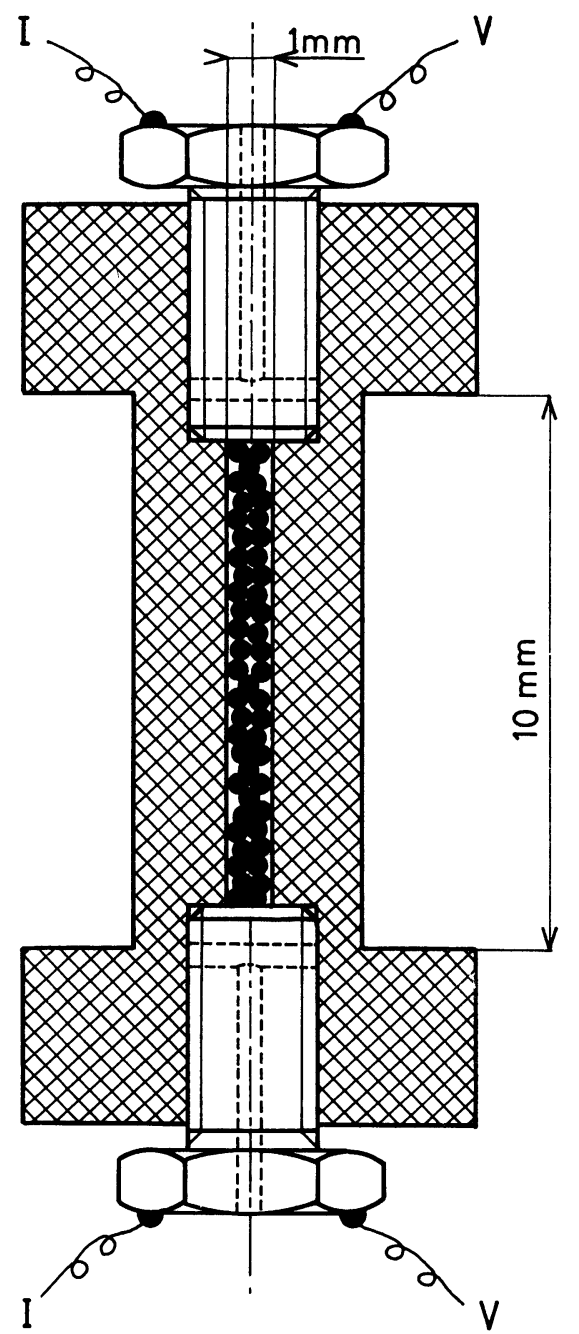

FIG. 1. - Typical granular sample. The grains $(170 \pm 10 \mu$ in diameter) are kept in position inside a plastic cylinder by two screws. These serve as electrical contacts and are conveniently drilled to allow the passage of liquid $\mathrm{He}$.

can be neglected. On the other hand, if there is a tunneling interaction between grains a temperature interval may exist where the Josephson free energy $\left|F_{\mathrm{J}}\right|<k T\left(F_{\mathrm{J}}=-(\hbar / 2 \mathrm{e}) I_{\mathrm{c}} \cos \varphi_{\alpha \alpha^{\prime}}\right.$, where $\varphi_{\alpha \alpha^{\prime}}=\varphi_{\alpha}-\varphi_{\alpha^{\prime}}, \alpha$ and $\alpha^{\prime}$ designing first neighbours), resulting in fluctuations of the phases $\varphi_{\alpha}$ while $I_{\mathrm{c}}=I_{\mathrm{c}}(|\Delta|)$ practically does not fluctuate. At temperatures close to $T_{\mathrm{c}}$ we expect no long-range correlation of the phases while a weak first-neighbour correlation $\eta=\left\langle\mathrm{e}^{i \varphi_{\alpha \alpha^{\prime}}}\right\rangle$ may be present. The representative vectors $\Delta_{\alpha}$ are oriented at random in the complex plane, as shown pictorially in figure 2 . This defines the paracoherent region. As temperature decreases below a certain coherence temperature $T_{0}$, where $\left|F_{\mathrm{J}}\right| \sim k T_{0}$, a phase transition to a coherent state characterised by the appearance of long-range correlations between the phases, is performed. It is natural to define an order parameter $M=\left\langle\mathrm{e}^{i \varphi_{\alpha}}\right\rangle=0$ in the coherent region, which may be taken as real $\left(M=<\cos \varphi_{\alpha}>\right)$ in the absence of applied fields and currents. We note that the average superconduct-
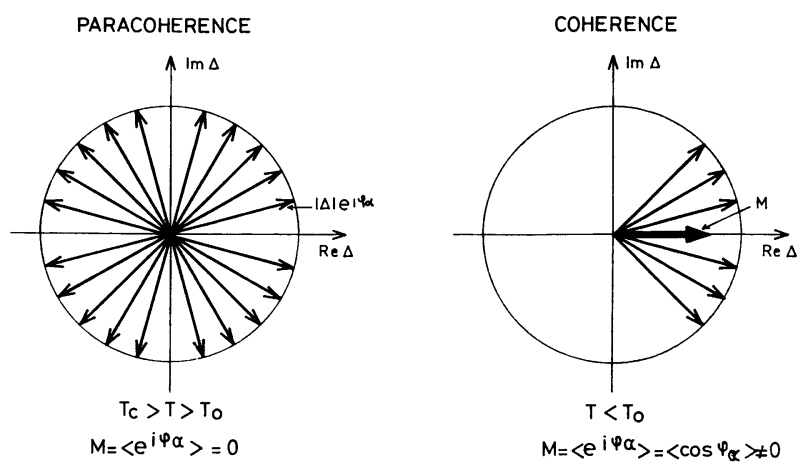

FIG. 2. - A pictorial description of phase ordering in the complex plane.

ing order parameter in the system $\langle\Delta\rangle=M|\Delta|$ is non zero only in the coherent region of temperatures.

More precisely, let $E_{k \alpha}$ design the unperturbed electronic energy levels of the $\alpha$ th grain. The partition function of the system is

$$
Z_{0}=\sum_{k} \exp \left(-\beta \sum_{\alpha} E_{k \alpha}\right)
$$

The phase in each grain can be assigned arbitrarily, any two phase configurations differing only by a gauge transformation. If now the tunneling Hamiltonian is switched on, second-order perturbation theory shows [9] that the energy levels become $E_{k \alpha}^{\prime}+\delta E_{k \alpha} \cos \varphi_{\alpha \alpha^{\prime}}$, where $E_{k \alpha}^{\prime}$ represents all phaseindependent terms. The partition function is transformed into

$$
Z^{\prime}=\sum_{k} \exp \left(-\beta \sum_{\alpha}\left(E_{k \alpha}^{\prime}+\sum_{\alpha^{\prime}}^{\prime} \delta E_{k \alpha} \cos \varphi_{\alpha \alpha^{\prime}}\right)\right),
$$

where the prime denotes summation over first neighbours only. If the perturbation is small, the phase dependent free energy difference becomes

$$
\begin{aligned}
\delta F_{\varphi} & =-k T \delta \ln Z_{0}^{\prime}=\sum_{\alpha<\alpha^{\prime}}^{\prime}<\delta E_{k \alpha}>_{0} \cos \varphi_{\alpha \alpha^{\prime}} \\
& =-J \sum_{\alpha<\alpha^{\prime}}^{\prime} \cos \varphi_{\alpha \alpha^{\prime}}
\end{aligned}
$$

where

$$
Z_{0}^{\prime}=Z^{\prime}\left(\delta E_{k \alpha} \rightarrow 0\right) \text { and } J=\frac{\hbar}{2 \mathrm{e}} I_{\mathrm{c}}
$$

is just the Josephson coupling energy. Now, expression (3) gives the free energy at constant phase. Actually, the phase configuration cannot be fixed in a granular sample and one has to allow for a statistical distribution of phases. The partition function becomes finally, for a system of $N$ grains with no applied current,

$$
\begin{aligned}
Z=Z_{0}^{\prime} Z_{\varphi}= & Z_{0}^{\prime}\left(\frac{1}{2 \pi}\right)^{N} \iint \cdots \int \times \\
& \times \exp \left(\beta J \sum_{\alpha<\alpha^{\prime}}^{\prime} \cos \varphi_{\alpha \alpha^{\prime}}\right) \prod_{\alpha=1}^{N} \mathrm{~d} \varphi_{\alpha}
\end{aligned}
$$


The main interest of expression (5) is to show, firstly that $Z$ can be factored into a phase-independent part $Z_{0}^{\prime}$ and a phase-dependent one $Z_{\varphi}$ and secondly, that $Z_{\varphi}$ coincides with the partition function of a classical ferromagnetic Heisenberg system with rigourously isotropic two-dimensional spin space (the complex plane). We expect indeed a phase transition of such a system to a coherent state, as described above.

We have obtained [5] the equations ur phase motion in a homogeneous system in the Bethe approximation [10], which amounts to consider a cluster whose central grain is subject to the first-neighbour interaction $J$ and whose outer grains are linked to the external world by some mean field $H_{1}$. The resulting equations of motion for a single junction in the cluster are

$$
\left.\begin{array}{rl}
\frac{\mathrm{d} \theta}{\mathrm{d} t} & =\frac{2 \mathrm{e}}{\hbar} V_{1}, \\
\frac{\hbar}{2 \mathrm{e}} C_{1} \frac{\mathrm{d}^{2} \theta}{\mathrm{d} t^{2}} & =I_{1}-\frac{2 \mathrm{e}}{\hbar}\left(\eta J+M H_{1}\right) \sin \theta- \\
-\frac{\hbar}{2 \mathrm{e}} \frac{1}{R_{1}} \frac{\mathrm{d} \theta}{\mathrm{d} t},
\end{array}\right\}
$$

where $\theta$ represents the departure of the phase from its equilibrium value, and $V_{1}, C_{1}, R_{1}$ and $I_{1}$ are the voltage, capacity, normal resistance and (assumedly small) current in the barrier, respectively. Eq. (6) are just the equations of phase motion of a single point contact whose maximum supercurrent would be given by

$$
<I_{\mathrm{c}}>=\frac{2 \mathrm{e}}{\hbar}<J>=\frac{2 \mathrm{e}}{\hbar}\left(\eta J+M H_{1}\right)
$$

and therefore displays a rather unusual temperature dependence [11]: the supercurrent per junction is weakened in the paracoherent region $T>T_{0}$ $\left(\eta<1, M=H_{1}=0\right)$ and strengthened in the coherent region $T<T_{0}$ as compared to that of a single junction.

3. dc properties of BGS. - According to the last paragraph, phase ordering should result in a sharp increase of the maximum supercurrent in the system. Above the coherence temperature and immediately below it, on the other hand, $\langle J\rangle$ is of the order of $k T$, and the effects of thermal noise cannot be neglected. In the strong damping approximation [8] noise produces a non zero dynamical resistance even at zero current bias,

$$
\left.\frac{R}{R_{\mathrm{q}}}\right|_{I \rightarrow 0}=I_{0}^{-2}\left(\frac{J}{k T}\right),
$$

where $R_{\mathrm{q}}$ is the quasiparticle resistance of the contact and $I_{0}$ is the modified Bessel function of order zero.
Performing the same analysis as in reference [8] on eq. (6) one obtains

$$
\left.\frac{R}{R_{\mathrm{q}}}\right|_{I \rightarrow 0}=I_{0}^{-2}\left(\frac{<J>}{k T}\right)
$$

for a bulk granular sample. For want of an exact calculation on the Heisenberg model, we have obtained $\langle J\rangle$ in terms of an Ising model in the Bethe approximation and compared our experimental results [5] with the predictions of eq. (9). This is shown in figure $3 a$. Of course, the use of an Ising model is objectionable in principle, because we are replacing the infinite degeneracy of the complex plane by the two-fold degeneracy of one-dimensional spin space. However, a look at figure $3 b$ shows that the devia-

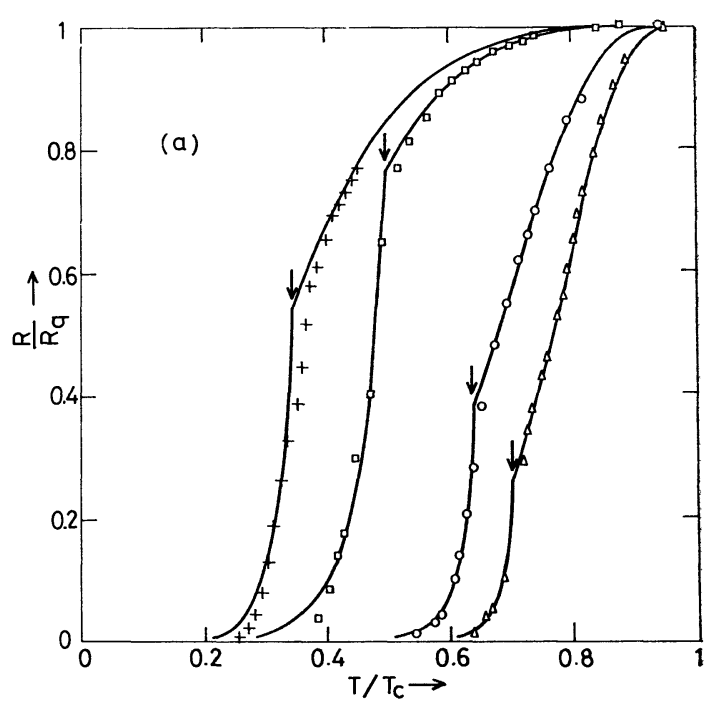

(a)

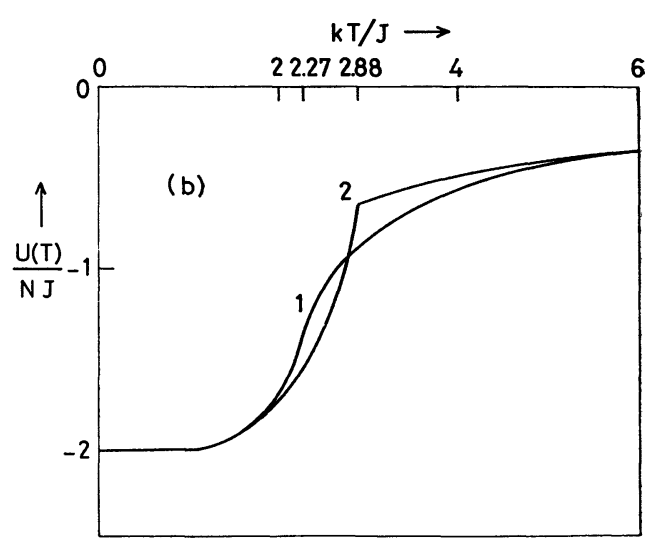

(b)

FIG. 3. - a) Experimental dynamical resistance $R$ at zero current bias divided by the quasiparticle resistance $R_{\mathrm{q}}$ of granular samples. Crosses : $\mathrm{Nb}, R_{\mathrm{q}}=13.0 \Omega$; squares : Ta, $R_{\mathrm{q}}=12.3 \Omega$, circles : Ta, $R_{\mathrm{q}}=3.4 \Omega$; triangles : Ta, $R_{\mathrm{q}}=0.89 \Omega$. Full curves are a fit from a calculation based on the phase transition model in the Bethe approximation. The arrows mark the coherence temperatures. (From ref. [5].) b) A comparison between the internal energy per particle from an exact solution of the Ising model (curve 1) for the quadratic lattice and the corresponding predictions of the Bethe approximation (curve 2). 
tions of the Bethe approximation from an exact solution of the Ising model are qualitatively of the same type as those of theory from experiment in figure $3 a$. In other words, this tends to prove that the lack of fit in certain regions (cf. rounding off of experimental curves as compared with the slope discontinuity of the theoretical ones at $T_{0}$ ) is due to the approximation used rather than to the description in terms of a phase transition.

4. Electrodynamics of BGS in the coherent state. Below the coherence temperature, phase ordering extends itself over a large number of grains. We may consider the system as a continuum at least in the long wavelength limit, i. e. for excitations with wavenumber $k \ll d^{-1}$, where $d$ is the distance between grains. (More precisely $d=n^{-1 / 3}$, where $n$ is the number of grains per unit volume.) In doing so, we shall necessarily overlook the original non linear properties of the individual junctions, which should become apparent in a treatment of short wavelength excitations.

4.1 BULK PROPERTIES. - External electric and magnetic fields induce, on the one hand Josephson currents, on the other a linear response which can be easily estimated. Consider, for example, the normal conductivity. On the average, only one third of the junctions carry a current, the other two thirds being branched in parallel with respect to the direction of current flow. If $q$ is the coordination number, there are $\frac{1}{2} q$ junctions per grain and therefore the current is divided among $q / 6$ junctions of resistance $R_{1}$ across a length $d$ and a section $d^{2}$. One obtains

$$
\sigma \simeq \frac{q}{6}\left(d R_{1}\right)^{-1}
$$

that is, for $d \sim 100 \mu, R_{1} \sim 1 \Omega$ the resistivity

$$
\sigma^{-1} \sim 10^{-2} \Omega . \mathrm{cm} \text {. }
$$

The dielectric constant can be obtained similarly [12] by computing the field and electric induction in the $q / 6$ junctions of capacity $C_{1}$ :

$$
\varepsilon=4 \pi \frac{q}{6} \frac{C_{1}}{d}
$$

which, for a typical capacity of $10^{-12} \mathrm{~F}$ gives values of $\varepsilon \sim 10^{3}$.

A magnetic field $\mathbf{H}$ applied on the system results in the diamagnetic response of the individual grains. The average field in the volume occupied by the BGS is the magnetic induction

$$
\mathbf{B}=\mathbf{H}+4 \pi \mathbf{M}=\mu \mathbf{H}
$$

where the magnetisation $\mathbf{M} \simeq-(\mathbf{H} / 4 \pi) f$ with $f$ the filling factor of the array. The permeability is then $\mu \simeq 1-f$. If the grains are perfect spheres, $\mu \sim 0.7$ for a diamond type structure $(q=4)$ and $\mu \sim 0.3$ for a close-packed array $(q=12)$. Taking into account eq. (11) one obtains for the characteristic impedance of the medium, in ohms,

$$
Z_{\mathrm{c}}=120 \pi \sqrt{\frac{\mu}{\varepsilon}} \simeq 10 \Omega
$$

4.2 SUPERCURRENTS AND FIELD EQUATIONS. In addition to circulating currents in each grain, Josephson supercurrents flow across the barriers. The resulting phase gradient along the sample implies that the phase order parameter $M=\left\langle\mathrm{e}^{i \varphi_{\alpha}}\right\rangle$ may no longer be real. Consider two neighbouring grains along the $z$-axis with (complex) superconducting order parameters $\Delta_{1}$ and $\Delta_{2}$. The supercurrent flowing through the junction is

$$
I_{\mathrm{s}}=I_{\mathrm{c}} \sin \left(\varphi_{1}-\varphi_{2}\right)=\frac{I_{\mathrm{c}} \operatorname{Im} \Delta_{1} \Delta_{2}^{*}}{|\Delta|^{2}}
$$

Making the continuum approximation, we replace $\mathrm{e}^{i \varphi_{1}}$ by $M, \mathrm{e}^{i \varphi_{2}}$ by $M+d \partial M / \partial z$. The $q / 6$ currentcarrying barriers per grain finally contribute to an average current density

$$
\begin{aligned}
\mathbf{j}_{\mathrm{s}}=- & \frac{q}{6} \frac{I_{\mathrm{c}}}{d} \times \\
& \times\left(\frac{1}{2 i}\left(M^{*} \nabla M-M \nabla M^{*}\right)+\frac{2|\mathrm{e}|}{\hbar c}|M|^{2} \mathbf{A}\right),
\end{aligned}
$$

where the last term, containing the vector potential $\mathbf{A}$, insures gauge invariance $\left({ }^{1}\right)$. This suggests the definition of a time and position dependent phase $\tilde{\varphi}=\frac{1}{2} i \ln M^{*} / M$. Eq. (15) becomes :

$$
\mathbf{j}_{\mathrm{s}}=-\frac{q}{6} \frac{I_{\mathrm{c}}}{d}|M|^{2}\left(\boldsymbol{\nabla} \tilde{\varphi}+\frac{2|\mathrm{e}|}{\hbar c} \mathbf{A}\right) .
$$

It is now an easy matter to write down the equations governing electromagnetic fields in BGS. From Josephson's voltage-frequency relation,

$$
\mathbf{E}=-\frac{1}{c} \frac{\partial}{\partial t}\left(\frac{\Phi_{0}}{2 \pi} \tilde{\nabla} \tilde{\varphi}+\mathbf{A}\right)
$$

where $\Phi_{0}$ is the flux quantum. The normal current density is $\mathbf{j}_{\mathbf{N}}=\sigma \mathbf{E}$ and the displacement current becomes $\mathbf{j}_{\mathrm{D}}=(\varepsilon / 4 \pi c) \partial \mathbf{E} / \partial t$. Finally, from Maxwell's equation

$$
\nabla \wedge \mathbf{B}=\frac{4 \pi \mu}{c}\left(\mathbf{j}_{\mathrm{s}}+\mathbf{j}_{\mathrm{D}}+\mathbf{j}_{\mathrm{N}}\right)
$$

(1) Note that the signs chosen in eq. (14) and (15) correctly give the direction of particle flow (toward increasing phases) and that (opposite) of electrical current. A different convention amounts to a description of Josephson effects in superconducting antimatter ! 
one obtains, for fields weak enough so that the vorticity of the phase gradient can be neglected,

$$
\nabla^{2} \mathbf{B}-\frac{4 \pi \sigma \mu}{c^{2}} \frac{\partial \mathbf{B}}{\partial t}-\frac{\mu \varepsilon}{c^{2}} \frac{\partial^{2} \mathbf{B}}{\partial t^{2}}=\frac{1}{\lambda^{2}} \mathbf{B}
$$

where

$\ddot{\lambda}=\left(\frac{\hbar c^{2} d}{(q / 6) 8 \pi \mu I_{\mathrm{c}}|M|^{2}}\right)^{1 / 2} \rightarrow \infty \quad$ as $T \rightarrow T_{0}$

is the penetration depth of a three-dimensional bulk granular superconductor. Similarly, one recognizes in eq. (19) a phase velocity of electromagnetic waves

$$
v=\frac{c}{\sqrt{\mu \varepsilon}}=c\left(\frac{d}{(q / 6) 4 \pi \mu C_{1}}\right)^{1 / 2} .
$$

Using the same typical parameters as above, one finds $v \sim 10^{-2} c$. It is interesting to compare these quantities with the corresponding ones $v_{\mathrm{J}}, \lambda_{\mathrm{J}}$, of a single barrier in the assembly :

$$
\frac{v}{v_{\mathrm{J}}}=\frac{\lambda}{\lambda_{\mathrm{J}}}=\frac{\sqrt{2 \lambda_{\mathrm{L}} d}}{L|M|} \sqrt{\frac{6}{q \mu}}
$$

where $L^{2}$ is the area of the junction and $\lambda_{L}$ the London penetration depth. With $|M| \sim 1, \lambda_{\mathrm{L}}=500 \AA$, $\lambda / \lambda_{\mathrm{J}} \sim 5$. As in a single junction there is a plasma frequency

$$
\omega_{0}=\frac{v}{\lambda}=\left(\frac{2 \mathrm{e} I_{\mathrm{c}}|M|^{2}}{\hbar c}\right)^{1 / 2},
$$

and a dispersion law for the propagation of electromagnetic waves of wave vector $\mathbf{k}$ and frequency $\omega$ is obtained from eq. (19) (neglecting the normal component of current) :

$$
\omega=\omega_{0}\left(1+k^{2} \lambda^{2}\right)^{1 / 2}
$$

which implies that only the propagation of waves with $\omega>\omega_{0}$ is possible in the medium. Contrary to the case of a single junction, propagation can take place in all directions.

The above equations describe a London superconductor with rather unusual characteristic lengths. In fact, eq. (16) and (17) give

$$
\mathbf{E}=\frac{4 \pi}{c} \lambda^{2} \frac{\partial \mathbf{j}_{\mathrm{s}}}{\partial t},
$$

which is the basic London equation. But $\lambda$ here may be of the order of a few millimeters! Parmenter [12] arrived at similar results by making the continuum assumption from the very beginning in the case of grains of a few angströms. This is equivalent to assuming that coherence is established simultaneously with the superconducting transition of the grains. Bulk grains, on the other hand, will always display a paracoherent behaviour in a finite temperature interval, although this can be made in principle as small as desired if one can manage to achieve a sufficiently strong coupling between grains.

4.3 QUANTUM INTERFERENCE. - As an application of the above formulae, let us consider a cylindrical sample of radius $R$, with a constant applied field along its axis. If the transversal dimensions of the sample are small compared to $\lambda$, the field $\mu H_{z}$ will be constant inside and derive from a vector potential $\mathbf{A}=\frac{1}{2} \mathbf{B} \wedge \mathbf{r}$. In a cylindrical system of coordinates $(r, \theta, z)$ the only component of $\mathbf{A}$ will be $A_{\theta}=\frac{1}{2} \mu r H_{z}$. Since there are no vortices $(R \ll \lambda), \nabla \wedge(\nabla \tilde{\varphi})=0$ and the gauge invariant phase gradient $\nabla \delta$ can be taken proportional to the vector potential in the London gauge

$$
\left.\nabla \delta\right|_{9}=\frac{1}{r} \frac{\partial \delta}{\partial \theta}=\frac{2 \pi}{\Phi_{0}} A_{\theta}=\frac{\pi}{\Phi_{0}} \mu r H_{z},
$$

and

$$
\delta=\frac{\pi}{\Phi_{0}} \mu r^{2} H_{z} \theta+\delta_{0}=\frac{\Phi}{\Phi_{0}} \theta+\delta_{0}
$$

where $\Phi$ is the total flux through the sample and $\delta_{0}$ is a constant such that $M_{0}=\left|M_{0}\right| \exp \left(i \delta_{0}\right)$ is the order parameter in zero field. Assume now that a current is applied along the $z$-axis. Because of the small dimensions of the sample $j_{z}$ is a constant and results in a constant $z$-component of the phase gradient. Straightforward integration over the sample cross section of $j_{z}$ from eq. (16) shows no interference effects on the total current. However, our derivation of eq. (16) assumes implicitly that $M$ does not change much over a large number of grains along directions perpendicular to the current flow. Eq. (27) shows that this need not be the case in the presence of the field. Furthermore, since the actual current paths do not necessarily coincide with the $z$-axis, each grain at an $r, \theta$ position receives current from others having different values of $r$ and $\theta$. In the extreme limit of very small $R$ we may tentatively replace $M$ in eq. (16) by its spatial average $\bar{M}$ in a plane $z=$ constant, which, taking into account eq. (27) is :

$$
\bar{M}=\left|M_{0}\right| \frac{\mathrm{e}^{i \delta_{0}}}{\pi R^{2}} \int_{-\pi}^{\pi} \int_{0}^{R} \exp \left(\frac{i \pi r^{2} B_{z} \theta}{\Phi_{0}}\right) r \mathrm{~d} r \mathrm{~d} \theta .
$$

One obtains

$$
\begin{aligned}
|\bar{M}| & =\left|M_{0}\right| \frac{\Phi_{0}}{\pi \Phi} \int_{0}^{\pi \Phi / \Phi_{0}} \frac{\sin x}{x} \mathrm{~d} x \\
& =\left|M_{0}\right| \frac{\Phi_{0}}{\pi \Phi} \quad \text { Si }\left(\frac{\pi \Phi}{\Phi_{0}}\right) .
\end{aligned}
$$

This results in small oscillations of $|\bar{M}|$ as shown in figure 4. In an extended sample one may expect a host of superimposed oscillations of different periodicity, corresponding to averages taken over diffe- 


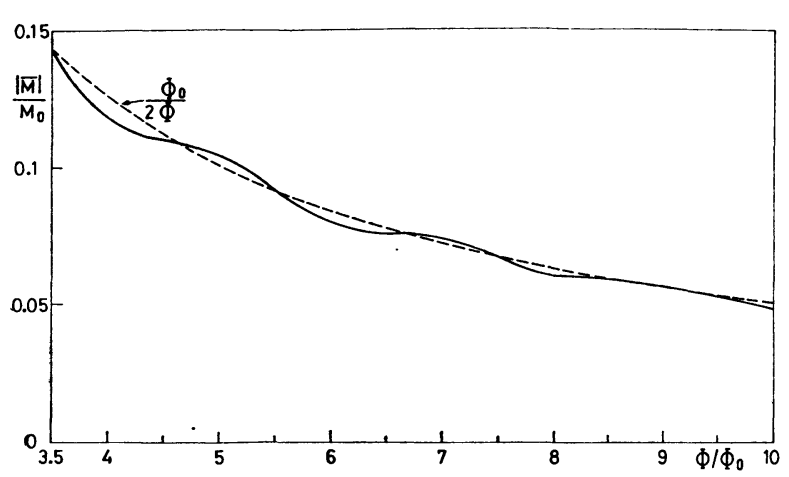

Fig. 4. - Average (over a plane normal to the applied field) of the reduced order parameter as a function of reduced applied flux (full curve). The asymptotic curve (dashed line) is shown for comparison.

rent values of $R$, but in any case of decreasing amplitude (cf. the $\left(\pi \Phi / \Phi_{0}\right)^{-1}$ factor in eq. (29)) as $R$ increases. We have indeed seen such oscillations in the critical supercurrent in our very first experiments [13]. The reason for their extremely small amplitude remained however a puzzle for some time. Actually, one observes three types of behaviour : well above $T_{0}$ there are no oscillations at all. As coherence gradually sets in, periodicities of the order of a few gauss appear, corresponding to areas of $\sim 1 \mu^{2}$. This obviously indicates a single junction interference pattern. Finally, below $T_{0}$ the multiply periodic pattern is observable.

5. Discussion. - We have studied statistical and electromagnetic properties of assemblies of Josephson barriers. Experimental and theoretical evidence indi- cates the existence of a phase transition between a paracoherent (disordered) and a coherent (ordered) state. This phase transition results from a first neighbour interaction between man-made objects (the grains). One may expect that these artificial systems will allow the experimental study of subjects such as critical indices in two- and three-dimensional structures, statistics of the few-body problem, etc. A drawback, of course, is that we cannot hope for the same degree of regularity as is found in natural crystals.

In the coherent state bulk granular superconductors behave as extreme type II superconductors with unusual parameters. In fact, one can estimate the coherence length at $T=0$ as being of the order of the distance between grains, while the penetration depth is of the order of a few millimeters. If one can extrapolate the definition of the GinsburgLandau parameter to this case, one finds values of $\kappa \sim 50$. Conversely, they can be regarded as superconducting " macrojunctions ", since they display the main features of weak superconductivity, such as quantum interference, plasma oscillations and radiation [2]. It is perhaps a little early to forecast any definite application of these systems : efforts should be made towards the fabrication of rugged and reproducible devices as well as towards a deeper theoretical understanding of their behaviour.

Acknowledgments. - Most of the experimental results reported here have been obtained by P. Pellan, G. Dousselin and, at an early stage, $\mathbf{H}$. Cortès. It is a pleasure to point out that without their results and the discussions that followed, this work would not have been possible.

\section{References}

[1] Cortès, H., Pellan, P. and Rosenblatt, J., Proc. 12th Int. Conf. on Low Temperature Physics (Keigaku Publishing Co., Tokyo) 1970, p. 487.

[2] Clark, T. D., Phys. Rev. B 8 (1973) 137, and preceding paper.

[3] Tilley, D. R., Phys. Lett. A 33 (1970) 205.

[4] Rosenblatt, J., Cortes, H. and Pellan, P., Phys. Lett. A 33 (1970) 143.

[5] Pellan, P., Dousselin, G., Cortès, H. and Rosenblatt, J., Solid State Commun. 11 (1972) 427.

[6] Clark, T. D. and Tilley, D. R., Phys. Lett. A 28 (1968) 62.

[7] Warman, I., Jahn, M. T. and KaO, Y. H., J. App. Phys. 42 (1971) 5194.
[8] Ambegaokar, V. and Halperin, B. I., Phys. Rev. Lett. 22 (1969) 1364.

[9] Anderson, P. W., Progr. Low Temp. Phys. 5 (1967) 1.

[10] Bethe, H. A., Proc. R. Soc. 216 (1935) 45.

[11] Pellan, P., Dousselin, G. and Rosenblatt, J., Revue Phys. Appl. 8 (1973) 471.

[12] Parmenter, R. H., Phys. Rev. 154 (1967) 353 and Phys. Rev. 167 (1968) 387.

[13] Quite recently a similar behaviour has been observed in granular films by Hansma, P. K., Solid State Commun. 13 (1973) 397. 